\title{
Prostate brachytherapy procedural training: incorporation of related procedures in resident training and competency assessment
}

\author{
Jennifer Bachand, B.S.', Samuel R. Schroeder, MD, MBA², Neil B. Desai, MD², Michael R. Folkert, MD, PhD² \\ IUT Southwestern Medical School, Simmons Comprehensive Cancer Center at the University of Texas Southwestern Medical Center, Dallas, TX USA \\ 2Department of Radiation Oncology, Simmons Comprehensive Cancer Center at the University of Texas Southwestern Medical Center, Dallas, \\ TX USA
}

\begin{abstract}
Purpose: Inadequate procedural training is of increasing concern in resident training, especially in prostate brachytherapy (PB). Transperineal rectal spacer placement (TRSP) requires many of the same proficiencies as PB. This work describes the assessment of teaching techniques focusing on developing critical competencies for PB using related clinical procedures (TRSP).

Material and methods: For PB and TRSP, key competencies were identified: 9 for PB and 7 for TRSP; 4 are shared between PB and TRSP. "Comfort level" with these procedures was assessed prior to and following participation in TRSP.

Results: 8 of 12 trainees at our institution participated in TRSP procedures. 2 of these trainees had prior experience with PB or related procedures and were excluded. Trainees self-reported "comfort levels" between 0 and 3 for four competency domains. Initial median comfort (MC) level for competency domains relevant to PB included: patient positioning (median 1, range 0-2), transrectal ultrasound imaging (median 1, range 0-1), fiducial placement (median 1 , range 0-1), and hydrodissection (median 0, range 0-1). Median number of TRSP procedures performed by assessed trainees during the analysis period was 4 (range 1-6). Following TRSP procedure training, MC level increased: 2 points for patient positioning (median 3 , range $1-3 ; p<0.01$ ), 1.5 points for transrectal ultrasound imaging (median 2.5, range 1.3, $p<0.001$ ); 1 point for fiducial placement (median score 2, range 1-3; $p<0.001$ ); and 1.5 points for hydrodissection (median score 2, range 1-3; $p<0.001$ ).

Conclusions: Increasing trainee involvement in related procedures to develop core competencies may help facilitate increased comfort with common skills critical to the independent performance of PB.

J Contemp Brachytherapy 2019; 11, 6: 601-606 DOI: https://doi.org/10.5114/jcb.2019.90984
\end{abstract}

Key words: prostate brachytherapy, rectal spacer, resident education, simulation.

\section{Purpose}

Utilization of brachytherapy for the management of localized prostate cancer is decreasing in the United States [1]. Studies using large cancer registries have explored these trends, including a study by Martin et al. using the National Cancer Database (NCDB) that identified a change in utilization from $17 \%$ in 2002 to $8 \%$ in 2010 [2], and a study by Mahmood et al. using the Surveillance, Epidemiology, and End Results (SEER) Program Registry that identified a decrease in brachytherapy procedures from $44 \%$ in 2004 to $38 \%$ in 2009 [3]. A more recent review of the SEER registry data demonstrated that the use of both monotherapy and combination brachytherapy in prostate cancer decreased by more than $60 \%$ from 2004 to 2013 [4].
This decrease in utilization contrasts with high quality data demonstrating the efficacy and favorable quality of life outcomes for patients treated with brachytherapy monotherapy, or as part of combined modality therapy with external beam radiation for high risk disease where significant biochemical progression-free-survival (bPFS) improvements have been demonstrated $[5,6,7]$. In light of the operator dependency of brachytherapy, however, cautionary data on increased toxicity of brachytherapy given as a boost to external beam radiation therapy (EBRT) or when sub-optimally applied $[8,9,10,11]$ have increased the focus on establishing high quality implant technique, and therefore training of radiation oncology residents.

The decreased utilization of prostate brachytherapy has reduced exposure to the procedure during residency 
training. For instance, Compton et al. reported a significant decline in per resident logged prostate interstitial brachytherapy cases between academic years (AY) 20062007 (2440 procedures total, 114 residents, 21.4 procedures/resident) and AY 2010-2011 (2326 procedures total, 129 residents, 18 procedures/resident) [12]. Continuing this trend, a survey of U.S. chief residents between 2013 and 2015 demonstrated very high rates of respondents noting no or an inadequate level of exposure (40-85\%) to prostate brachytherapy procedures (low dose rate-LDR, or high dose rate - HDR) [13].

The "see one, do one, teach one" principle of procedure education that dates back to Halsted is one of the foundations of surgical and interventional procedure training, but without adequate exposure to a critical supply of cases, residents will not be comfortable with the procedure and will be less likely to perform it as a practitioner. The quality of brachytherapy implantation is also likely to be impacted even for those engaged in the practice of brachytherapy, as low procedural volume has also been shown to impact outcomes [14]. Currently, the Accreditation Council for Graduate Medical Education (ACGME) requires a minimum of five interstitial brachytherapy cases (all disease sites combined) to graduate Radiation Oncology residency; however, this minimum requirement is likely insufficient, as many practicing physicians do not feel comfortable enough with their proficiency in the procedure to establish their own brachytherapy practice, and the interstitial case requirement may be satisfied without even performing a single prostate brachytherapy case. Furthermore, given the decreased utilization of brachytherapy described above, access to cases is limited and residents may need to find other ways to increase their exposure to procedural skills necessary to perform brachytherapy treatment.

In the report by Compton et al., it was recommended that the community interested in training residents in brachytherapy should "consider methods to ensure that residents obtain sufficient experience in the future" [12]. Prostate brachytherapy is a complex, multi-step procedure that requires expertise in multiple underlying technical skills, or 'competencies', to perform safely and effectively. Gallagher et al. discussed the concept of skill acquisition through skills generalization and skill transfer [15], and it may be possible to develop competencies necessary for prostate brachytherapy by performing other procedures used in the radiation oncology clinic. Transperineal rectal spacer placement (TRSP) is one such procedure, where a material is injected transperineally under ultrasound guidance into the potential space between the rectum and prostate prior to radiation therapy for prostate cancer to increase separation between the structures and reduce incidental radiation dose delivery to the rectum [16]. There is substantial overlap in the generalizable skills or competencies between prostate brachytherapy and TRSP procedure domains, such as patient positioning, transrectal ultrasound imaging, needle placement, and complications management. Thus, mastering fundamental competencies in the TRSP domains may benefit performance of prostate brachytherapy.
In order to better characterize trainee competence, prior studies in internal medicine have used resident "comfort level" (ranging from 1 - very uncomfortable to 5 - very comfortable performing the procedure) in conjunction with minimum requirements established by the ACGME [17]. To better clarify competence for specific types of interstitial brachytherapy, trainee comfort could be used to assess critical procedural skills. This work describes the assessment of teaching techniques focusing on performance of key procedure competencies that are shared with interstitial prostate brachytherapy (PB) and transperineal rectal spacer placement (TRSP).

\section{Material and methods}

For PB and TRSP, key competencies were identified; 8 for PB and 7 for TRSP (Table 1). Four of these procedures (patient positioning, transrectal ultrasound imaging, needle placement, and complications management) are shared between PB and TRSP. Fiducial placement is a needle placement procedure that may actually be identically performed in both PB and TRSP; placement of needles for hydrodissection and spacer placement in TRSP is felt to be analogous to needle placement in PB as well.

The placement of TRSP has been described previously [18]. At our institution, we use an FDA-approved polyethylene glycol hydrogel (SpaceOAR; Augmenix, Inc., Bedford, MA USA; now Boston Scientific, Inc.). Briefly, the procedure takes place either in a clinic setting with local anesthesia/moderate sedation or in the operating room under general anesthesia per patient preference or if clinic placement cannot be tolerated. Prior to the procedure, the patient is instructed to perform an enema. During the procedure, the patient is placed in the dorsal lithotomy position and the transrectal ultrasound (TRUS) is placed with a stepper for visualization. In the clinic setting, local anesthesia is used in the subcutaneous perineal area and bilateral neurovascular bundles, and moderate sedation with intravenous midazolam and fentanyl is provided; in the operating room setting, the patient is placed under general anesthesia and no local anesthesia is used. Gold fiducial seed markers are then placed with a transperineal approach. An 18 gauge needle is then inserted with the transperineal approach and guided to Denonvilliers' fascia where hydrodissection takes place. Once adequate separation between the rectum and prostate is visualized on ultrasound, the injectable spacer gel is introduced (Figure 1).

Prior to involvement in TRSP procedures, the attending reviews the competency steps involved and relevant ultrasound anatomy. Use of prostate phantoms (CIRS Model 053S Tissue Equivalent Prostate Phantom; Computerized Imaging Reference Systems, Incorporated, Norfolk, VA USA) provided initial exposure to ultrasound setup, imaging, and needle visualization and placement (Figure 2) following the method of Thaker et al. [19]. For the first patient, the attending demonstrates the procedure in full with the resident observing; subsequent procedures incorporate direct resident involvement.

Trainee involvement in TRSP has been implemented at our institution for approximately 12 months, and 
Table 1. Core competencies for transperineal rectal spacer placement (TRSP) and prostate brachytherapy (PB) Procedure domains competency

\begin{tabular}{cc}
\hline Rectal spacer competency & Prostate brachytherapy competency \\
\hline no direct equivalent & Patient selection \\
Patient positioning for endocavitary procedures & Patient positioning for endocavitary procedures \\
Transrectal ultrasound placement and operation & Transrectal ultrasound placement and operation \\
Administration of local anesthesia & no direct equivalent \\
no direct equivalent & Treatment planning \\
Fiducial seed placement & Needle placement \\
Hydrodissection & Needle placement \\
no direct equivalent & Source delivery \\
no direct equivalent & Post-implant dosimetry \\
Rectal spacer placement & Needle placement \\
Post-procedure complications management & Post-procedure complications management
\end{tabular}
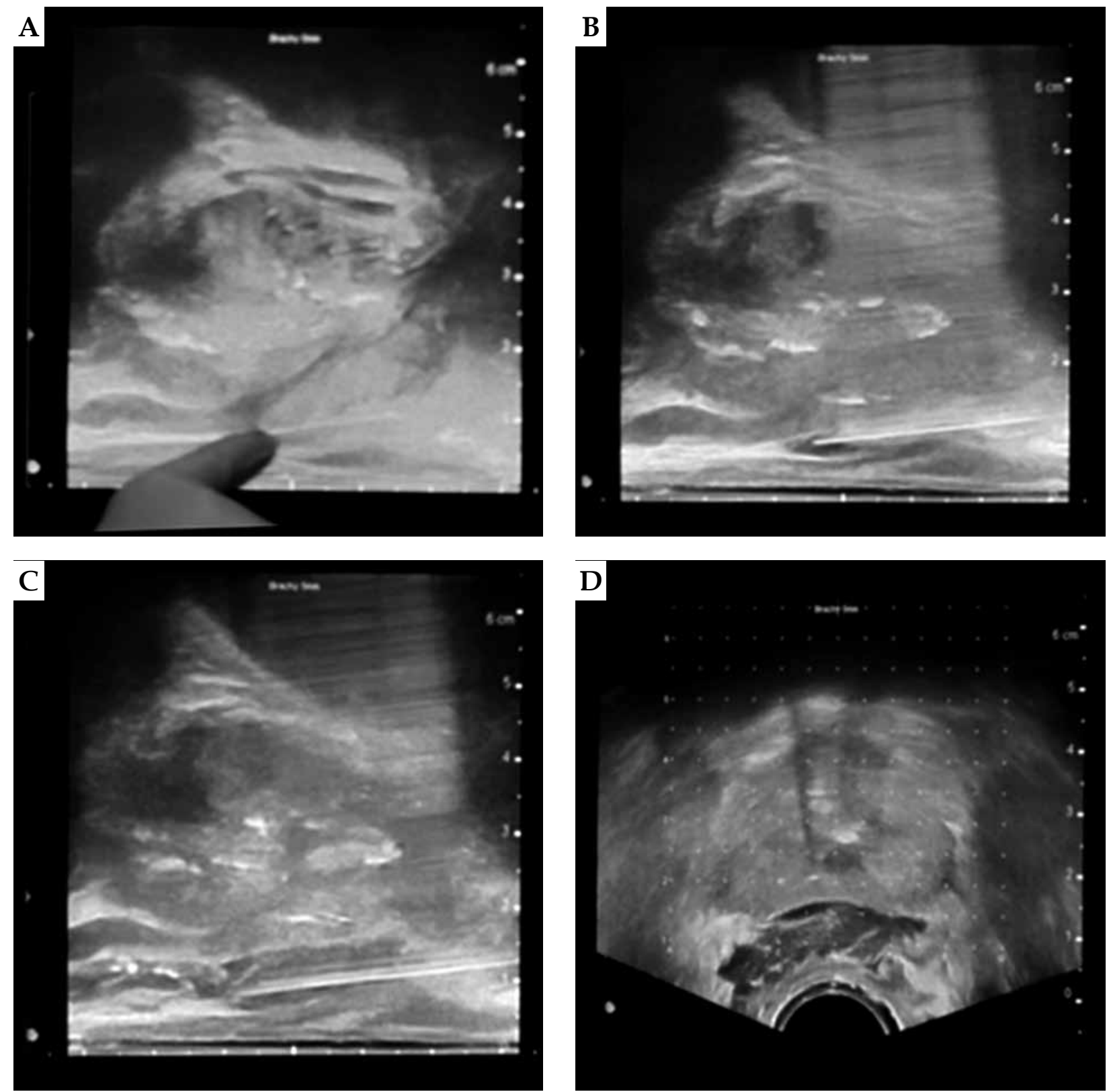

Fig. 1. Transperineal spacer placement; A) identification of rectoprostatic space on transrectal ultrasound; B) needle placement and hydro-dissection; C) rectal spacer insertion; D) rectal spacer material in place between prostate and rectum (axial view) 


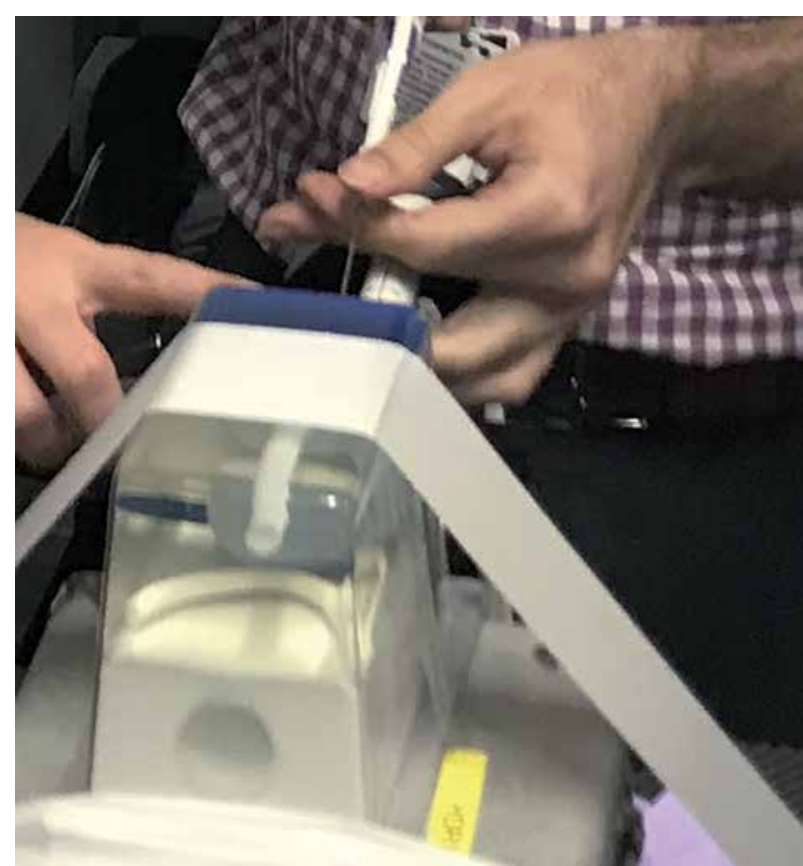

Fig. 2. Use of tissue equivalent phantom for initial prostate procedure training

"comfort level" with these procedures (as measured on a Likert scale, Table 2) was assessed prior to and following participation in these procedures. "Comfort level" was graded on a scale of $0-3$, with 0 corresponding to the resident having no comfort at all with the procedure, to 3 corresponding to the resident being comfortable with performing the procedure completely independently. Trainees were also asked how many procedures they felt (in their opinion) they would need to perform to reach independent competency. Statistical comparisons were

Table 2. "Comfort level" scale used to assess trainee performance

"Comfort level" assessment

0 not comfortable at all

1 comfortable with direct, hands on supervision

2 comfortable with attending present offering suggestion/ad-

vice only

3 comfortable doing independently performed using IBM SPSS Statistics v.25.0 (IBM, New York, NY USA); "comfort level" comparisons were performed using the paired sample $t$-test.

\section{Results}

Over a 12-month period, 8 of 12 trainees at our institution participated in TRSP procedures. Two of these trainees had prior experience with prostate brachytherapy or other endorectal procedures and were excluded from further analysis. Prior to performing TRSP procedures, median comfort level for competency domains relevant to prostate brachytherapy included: patient positioning (median 1, range 0-2); transrectal ultrasound imaging (median 1, range 0-1); needle placement domains: fiducial placement (median 1, range 0-1); hydrodissection (median 0 , range $0-1$ ) (Table 3).

Median number of TRSP procedures performed by assessed trainees during the analysis period was 4 (range 1-6). Following TRSP procedure training, median comfort level increased by 2 points for patient positioning (median 3 , range $1-3 ; p<0.01$ ) and by 1.5 points for transrectal ultrasound imaging (median 2.5, range 1-3; $p<0.001$ ). Needle placement domains improved by 1 point for fiducial placement (median score 2, range $1-3 ; p<0.001$ ) and by 1.5 points for hydrodissection (median score 2, range 1-3; $p<0.001$ ).

No trainees felt that they were ready to perform all competencies for TRSP independently at the end of the evaluation time point, responding that a median of 5 additional cases overall would be necessary for "independent" competency (Table 4 ). However, $66.7 \%$ of residents felt that they were fully independently competent in patient positioning, and $50 \%$ felt that they were fully independently competent in ultrasound use and local anesthesia. $33.3 \%$ felt that they were fully independently competent in fiducial placement and hydrodissection. Of note, in all cases where the resident stated that they had reached the "independent" comfort level in a competency, their performance in that competency was noted by the observing brachytherapy procedure attending to be satisfactory. As directly observed by brachytherapy procedure attendings during subsequent prostate brachytherapy cases, those residents who had reached the "independent" comfort level for procedure skills in patient positioning and transrectal ultrasound use were able to perform those skills independently for PB pro-

Table 3. "Comfort level" assessment of trainees before and after transperineal rectal spacer placement (TRSP) procedure training

\begin{tabular}{lcccc} 
TRSP procedure & \multicolumn{2}{c}{ Prior to training } & \multicolumn{2}{c}{ Following training } \\
\cline { 2 - 5 } & Median comfort level & Range & Median comfort level & Range \\
\hline Patient positioning & 1 & $0-2$ & 3 & $1-3(p<0.01)$ \\
\hline Transrectal ultrasound imaging & 1 & $0-1$ & 2.5 & $1-3(p<0.001)$ \\
\hline \begin{tabular}{l} 
Needle placement domains: \\
\hline Fiducial placement
\end{tabular} & 1 & $0-1$ & 2 & $1-3(p<0.001)$ \\
\hline Hydrodissection & 0 & $0-1$ & 2 & $1-3(p<0.001)$
\end{tabular}


cedures. All $(100 \%)$ of the residents felt that procedure education with TRSP placement had a positive impact on their confidence and competencies with transperineal prostate brachytherapy procedures.

\section{Discussion}

Achieving clinical competency in core procedures relevant to the practice of radiation oncology is a primary concern for residency education. Numerous studies have shown that in prostate brachytherapy, implant quality is critical to achieve optimal outcomes as biochemical progression-free survival is significantly linked to the dose received by $90 \%$ of the prostate volume $\left(\mathrm{D}_{90}\right)[20,21]$, and urinary toxicities are also closely associated with urethral dosimetric quantities [22]. Dosimetric guidelines have been provided by the American Brachytherapy Society (ABS) and the American Association of Physicists in Medicine (AAPM) $[23,24,25]$.

Given the technical nature of seed placement and the importance of implant dosimetry, decreased exposure to prostate brachytherapy during residency is concerning and may deter physicians from offering brachytherapy as a treatment option. Additionally, decreased brachytherapy experience may be dangerous to patients as those treated by higher volume physicians were found to have a lower risk of recurrence and a lower risk of prostate cancer death $[6,11]$. Furthermore, reporting requirements specified by the Nuclear Regulatory Commission may further dissuade providers from offering brachytherapy as minor underdosing can be classified as a reportable error [1], making brachytherapy a potential liability. Finally, the shift away from brachytherapy to newer and more expensive therapies places a significant financial burden on the healthcare system and patients. In the future, development of alternative payment models may incentivize brachytherapy because of its efficient and cost-effective nature, further emphasizing the importance of prostate brachytherapy experience during residency training. Lastly, with data suggesting meaningful benefits to combination brachytherapy boost, where toxicity is increased, an increased proportion of $\mathrm{PB}$ in the future is likely to require a mix of HDR/LDR skill sets and harbor a narrower therapeutic margin in terms of operator competency effect on treatment toxicity.

Thus, high quality procedural education and training is of the utmost importance for patient care and safety, for physician efficacy, and for the economic impact of our increasingly robust medical management of cancer. As the use of brachytherapy declines, it is important to integrate the technical skills involved with the procedure into resident education and to ensure that enough repetitions occur so that the trainees feel comfortable performing certain key competencies. Our observed increase in trainee comfort levels across all categories of assessed TRSP procedure components indicates that simple component training could be an important aspect of all radiation oncology training programs. With mastery of some key components, residents will be able to focus on developing overall higher comfort levels with other aspects of prostate brachytherapy procedures, which in turn could help to counteract downward trends in the use of prostate
Table 4. Range of self-reported additional cases necessary for trainees to reach "independent" competency in transperineal rectal spacer placement (TRSP) procedures

\begin{tabular}{ll} 
TRSP procedure & Range \\
\hline Patient positioning & $1-6$ \\
\hline Transrectal ultrasound imaging & $3-6$ \\
\hline Needle placement domains: & \\
\hline Fiducial placement & $3-10$ \\
\hline Hydrodissection & $4-10$
\end{tabular}

brachytherapy as trainees gain the confidence to perform the necessary procedures. More importantly, prostate brachytherapy could become an even safer and more effective prostate cancer therapy option as increased physician comfort and subsequent increased experience lead to lower recurrence rates and lower prostate cancer death risk. This is supported by similar work in gynecologic brachytherapy, where proficiency-based training was recently reported by Zhao et al.; they showed that simulation training in the setting of cervical cancer brachytherapy showed improvements in resident performance [26].

There are several limitations of the study, primarily sample size. Only six trainees were studied, but the ease of implementing the training and the simplicity and expediency of the surveys indicate that this evaluation could be replicated on a larger scale or even at a prostate brachytherapy-specific school such as the American Brachytherapy Society Prostate Brachytherapy School. The assessment of comfort level itself is limited; prior studies in internal medicine using resident "comfort level" to assess common procedural skills noted that resident comfort did not necessarily correlate to completion of ACGME requirements (for some procedures, comfort was achieved before meeting requirements, while it was not achieved until far exceeding requirements for others) [17]. Another limitation of the study is that it only assesses comfort levels in specific competency domains shared by PB and TRSP. Evaluating global improvements in the ability to deliver PB is well beyond the scope of this study, and we would recommend implementing this study on a larger scale with more participants and including evaluation of comfort levels during a prostate brachytherapy procedure itself before and after TRSP procedure training. Requiring more repetitions per trainee could also produce more robust results, as trainees indicated that they would need more cases in order to reach "independent" competency (Table 4). The study could also be expanded to include other specialized procedures involving these same TRSP components. This could be easily implemented at any institution with a high-volume radiation oncology center and physicians who are proficient in such procedures.

\section{Conclusions}

Optimally, all radiation oncology trainees will be exposed to sufficient prostate brachytherapy volume to 
be comfortable with all skills relevant to the procedure prior to entering independent practice, but this is currently not the case for many graduates. Increasing trainee involvement in related procedures that allow development of shared core competencies may help facilitate increased comfort with critical procedural skills. Overall, skill acquisition and transfer from TRSP could improve patient care and encourage the utilization of prostate brachytherapy.

\section{Disclosure}

The authors' institution received an unrestricted research grant from Augmenix, Inc. (Bedford, MA USA; now Boston Scientific, Inc.) for a separate project and non-financial support in the form of injectable rectal spacer material was provided by the same company for patients enrolled on two prospective trials in which residents participated in their care. Dr. Folkert has received unrestricted grant funding from Augmenix, Inc. (Bedford, MA USA; now Boston Scientific, Inc.) for an unrelated project, and Dr. Desai has received clinical trial funding from Augmenix, Inc. (Bedford, MA USA; now Boston Scientific, Inc.). The paper did not receive financial support from any source, nor was it supported by any grants; no author received any financial compensation for this work.

\section{References}

1. Petereit DG, Frank SJ, Viswanathan AN et al. Brachytherapy: where has it gone? J Clin Oncol 2015; 33: 980-982.

2. Martin JM, Handorf EA, Kutikov A et al. The rise and fall of prostate brachytherapy: use of brachytherapy for the treatment of localized prostate cancer in the National Cancer Data Base. Cancer 2014; 120: 2114-2121.

3. Mahmood U, Pugh T, Frank S et al. Declining use of brachytherapy for the treatment of prostate cancer. Brachytherapy 2014; 13: 157-162.

4. Chen J, Oromendia C, Halpern JA, Ballman KV. National trends in management of localized prostate cancer: A population based analysis 2004-2013. Prostate 2018; 78: 512-520.

5. Morris WJ, Tyldesley S, Rodda S et al. Androgen Suppression Combined with Elective Nodal and Dose Escalated Radiation Therapy (the ASCENDE-RT Trial): an analysis of survival endpoints for a randomized trial comparing a lowdose-rate brachytherapy boost to a dose-escalated external beam boost for high- and intermediate-risk prostate cancer. Int J Radiat Oncol Biol Phys 2017; 98: 275-285.

6. Hoskin PJ, Rojas AM, Bownes PJ et al. Randomised trial of external beam radiotherapy alone or combined with highdose-rate brachytherapy boost for localised prostate cancer. Radiother Oncol 2012; 103: 217-222.

7. Kishan AU, Cook RR, Ciezki JP et al. Radical prostatectomy, external beam radiotherapy, or external beam radiotherapy with brachytherapy boost and disease progression and mortality in patients with Gleason score 9-10 prostate cancer. JAMA 2018; 319: 896-905.

8. Guinot JL, Ricos JV, Tortajada MI et al. Comparison of permanent (125)I seeds implants with two different techniques in 500 cases of prostate cancer. J Contemp Brachytherapy 2015; 7: 258-264.

9. Chan EK, Keyes M, Pickles T et al. Decline in acute urinary toxicity: a long-term study in 2011 patients with prostate brachytherapy within a provincial institution. Brachytherapy 2014; 13: 46-52.
10. Spratt DE, Carroll PR. Optimal radical therapy for localized prostate cancer: recreation of the self-fulfilling prophecy with combination brachytherapy? J Clin Oncol 2018; 36: 2914-2917.

11. Chen AB, D'Amico AV, Neville BA et al. Provider case volume and outcomes following prostate brachytherapy. J Urol 2009; 181: 113-118; discussion 118.

12. Compton JJ, Gaspar LE, Shrieve DC et al. Resident-reported brachytherapy experience in ACGME-accredited radiation oncology training programs. Brachytherapy 2013; 12: 622-627.

13. Nabavizadeh N, Burt LM, Mancini BR et al. Results of the 2013-2015 association of residents in radiation oncology survey of chief residents in the United States. Int J Radiat Oncol Biol Phys 2016; 94: 228-234.

14. Luft HS, Bunker JP, Enthoven AC. Should operations be regionalized? The empirical relation between surgical volume and mortality. N Engl J Med 1979; 301: 1364-1369.

15. Gallagher AG, Ritter EM, Champion H et al. Virtual reality simulation for the operating room: proficiency-based training as a paradigm shift in surgical skills training. Ann Surg 2005; 241: 364-372.

16. Mariados N, Sylvester J, Shah D et al. Hydrogel spacer prospective multicenter randomized controlled pivotal trial: dosimetric and clinical effects of perirectal spacer application in men undergoing prostate image guided intensity modulated radiation therapy. Int J Radiat Oncol Biol Phys 2015; 92: 971-977.

17. Hicks CM, Gonzalez R, Morton MT et al. Procedural experience and comfort level in internal medicine trainees. J Gen Intern Med 2000; 15: 716-722.

18. Hatiboglu G, Pinkawa M, Vallée J-P, Hadaschik B, Hohenfellner M. Application technique: placement of a prostate-rectum spacer in men undergoing prostate radiation therapy. BJU Int 2012; 110: E647-E652.

19. Thaker NG, Kudchadker RJ, Swanson DA et al. Establishing high-quality prostate brachytherapy using a phantom simulator training program. Int J Radiat Oncol Biol Phys 2014; 90: 579-586.

20. Kattan MW, Potters L, Blasko JC et al. Pretreatment nomogram for predicting freedom from recurrence after permanent prostate brachytherapy in prostate cancer. Urology 2001; 58: 393-399.

21. Zelefsky MJ, Kuban DA, Levy LB et al. Multi-institutional analysis of long-term outcome for stages T1-T2 prostate cancer treated with permanent seed implantation. Int $J$ Radiat Oncol Biol Phys 2007; 67: 327-333.

22. Hathout L, Folkert MR, Kollmeier MA et al. Dose to the bladder neck is the most important predictor for acute and late toxicity after low-dose-rate prostate brachytherapy: implications for establishing new dose constraints for treatment planning. Int J Radiat Oncol Biol Phys 2014; 90: 312-319.

23. Davis BJ, Horwitz EM, Lee WR et al. American Brachytherapy Society consensus guidelines for transrectal ultrasound-guided permanent prostate brachytherapy. Brachytherapy 2012; 11: 6-19.

24. Nath R, Bice WS, Butler WM et al. AAPM recommendations on dose prescription and reporting methods for permanent interstitial brachytherapy for prostate cancer: report of Task Group 137. Med Phys 2009; 36: 5310-5322.

25. Yu Y, Anderson LL, Li Z et al. Permanent prostate seed implant brachytherapy: report of the American Association of Physicists in Medicine Task Group No. 64. Med Phys 1999; 26: 2054-2076.

26. Zhao S, Francis L, Todor D, Fields EC. Proficiency-based cervical cancer brachytherapy training. Brachytherapy 2018; 17: 653-659. 